\title{
Is inflammation good, bad or irrelevant for skeletal muscles in COPD?
}

\author{
Don D Sin, ${ }^{1,2}$ W Darlene Reid ${ }^{1,3}$
}

Skeletal muscle weakness is a common and serious finding in patients with advanced chronic obstructive pulmonary disease (COPD) and contributes to their morbidity and mortality, increasing the risk of exacerbations, hospitalisations and death by 3-4-fold. ${ }^{12}$ The treatment for muscle dysfunction of COPD is extremely limited, and the multitude of interventions to address poor muscle performance has not been fully explored in these patients. Over the past decade, there has been an explosion of interest and research on this topic. Despite that, the pathophysiological mechanisms linking the lung disease of COPD with skeletal muscle dysfunction remains largely unknown. Identifying a link between lung disease and muscle performance might indeed be a daunting task because of the influence of other comorbid conditions (and medications), previous musculoskeletal injury and the history of physical activity that might influence the current status of skeletal muscle in this condition. There are, however, several observations that are widely known and accepted. Firstly, skeletal muscle weakness increases with progression of disease. Secondly, histologically, biopsies of large limb muscles consistently demonstrate a reduction in muscle mass, especially of the anaerobic type-IIx fibres, a shift of fibre type from type 1 fibres to a predominance of type 2 fibres and the depletion of mitochondrial oxidative enzymes leading to uncoupling of oxidative phosphorylation and reduced aerobic capacity. ${ }^{3}$ Interestingly, these muscles also demonstrate increased oxidative stress and accelerated apoptosis and several recent reports have linked

\footnotetext{
${ }^{1}$ James Hogg iCAPTURE Center for Cardiovascular and Pulmonary Research, St Paul's Hospital, The University of British Columbia, Vancouver, BC, Canada; ${ }^{2}$ Department of Medicine (Respiratory Division), The University of British Columbia, Vancouver, BC, Canada; ${ }^{3}$ Department of Physical Therapy, The University of British Columbia, Vancouver, BC, Canada

Correspondence to: Dr D D Sin, James Hogg iCAPTURE Center for Cardiovascular and Pulmonary Research, St Paul's Hospital, Room \#368A, 1081 Burrard Street, Vancouver, BC V6Z 1Y6, Canada; dsin@mrl.ubc.ca
}

systemic inflammation with the physiological and histological changes observed in the skeletal muscles of COPD patients, raising the possibility that inflammation may be the principal "driver" of the muscle dysfunction in COPD. ${ }^{45}$ The latter concept has been fuelled by animal experiments, which showed that lung inflammation via systemic inflammation can induce local inflammatory responses in skeletal muscles, leading to muscle atrophy and inhibition of muscle regeneration. ${ }^{6}$

The study by Barreiro and colleagues ${ }^{7}$ in this issue of Thorax reports some provocative data that challenges the "inflammatory theory" of skeletal muscle dysfunction in COPD (see page 100).? In this carefully conducted cross sectional study, the authors found that there were less inflammatory changes in the quadriceps of patients with COPD than in the control subjects. In fact, protein expression in the muscles for tumour necrosis factor (TNF) $\alpha$ in COPD subjects was only about $60 \%$ of that in control subjects. $\mathrm{TNF} \alpha$ expression correlated positively with muscle function (ie, higher levels were associated with increased quadriceps strength). Other biomarkers of inflammation including vascular endothelial growth factor and TNF receptors showed similar trends. On the other hand, markers of oxidative stress were more prominent in COPD muscles. There was increased expression for protein carbonyl products in COPD muscles, which was associated with reduced quadriceps strengths. Collectively, these data raise the provocative hypothesis that local inflammation may not be detrimental (but may even be helpful) in maintaining skeletal muscle integrity and function of COPD patients.

Under the traditional paradigm, proinflammatory molecules such as TNF $\alpha$ are considered to be catabolic, leading to protein and muscle breakdown. However, there is emerging evidence that they may be anabolic in certain cases. For instance, $\mathrm{TNF} \alpha$ may play a critical regulatory role in muscle regeneration, possibly by activating the p38 mitogen activated protein kinase pathway, which is essential for myogenesis, ${ }^{8}$ and exogenous systemic administration of TNF $\alpha$ has been shown to promote skeletal muscle protein synthesis. ${ }^{9}$ Moreover, following acute injury to peripheral muscles, inflammatory cytokines are essential to muscle recovery and regeneration. ${ }^{10}$ Inflammatory cytokines such as TNF $\alpha$ can function both as a catabolic and an anabolic promoter, depending on its local concentration. At low concentrations (eg, $1 \mathrm{U} / \mathrm{ml}$ or less), TNF $\alpha$ is catabolic but at high concentrations, it becomes anabolic (eg, $100 \mathrm{U} / \mathrm{ml}$ or more). ${ }^{11}$

It is plausible then that in mild to moderate COPD, there may be increased local expression of inflammatory mediators in response to increased oxidative load (which may be helpful in maintaining muscle function) but with progression of disease resulting in progressive atrophy of peripheral muscles from disuse and medications (eg, systemic corticosteroids) and with additional increases in local oxidative stress, the local inflammatory responses may attenuate. This, in turn, may promote muscle breakdown and exacerbate skeletal muscle dysfunction. This concept may explain some of the discrepancies between the findings by Barreiro and colleagues ${ }^{7}$ and those by Montes de Oca et al, ${ }^{12}$ who studied a group of patients with COPD with milder disease (mean forced expiratory volume in $1 \mathrm{~s}, 43 \%$ of predicted versus $33 \%$ of predicted for Barreiro's study). Under this paradigm, reducing local or even systemic inflammation is unlikely to be helpful; it may even exacerbate muscle dysfunction.

Obviously, based on this cross sectional study, it is impossible to ascribe causation, and the hypothesis that local inflammation is a beneficial response to increased oxidative stress is still very speculative. Nevertheless, the findings of Barreiro et al suggest that we need to look at inflammation (at least in local tissues) in a new light. This is already happening in congestive heart failure (CHF). In CHF, where skeletal muscle changes are strikingly similar to those observed in COPD, ${ }^{13}$ several recent randomised controlled trials of anti-TNF therapies in CHF have failed to demonstrate any measurable benefits for the patient. In fact, two studies were terminated prematurely because of futility ${ }^{14}$ and the other alarmingly showed increased risk of death and hospitalisation for heart failure in patients randomised to receive infliximab. ${ }^{15}$ Previous studies examining the use of anti-inflammatory 
agents for soft tissue injury have demonstrated parallel findings. Although nonsteroidal anti-inflammatory drugs can reduce a particular marker of inflammation, their overall benefit towards healing of tendon and muscle injury is questionable. ${ }^{16}$

Skeletal muscle dysfunction is a major contributor of morbidity and mortality in COPD. Its pathogenesis, although multifactorial, is not clearly defined. The findings of Barreiro and colleagues ${ }^{7}$ challenge the prevailing notion that local inflammation contributes to the decline in muscle function. Like any good research, they have made us think outside the box and to approach this growing problem in a new and refreshing way. Only well designed clinical and animal studies in the future can answer the question: is inflammation good, bad or irrelevant for skeletal muscles in COPD? Or perhaps the more pressing question should be: what aspects of the inflammatory response should be adjusted during the various degenerative and regenerative phases of skeletal muscle as this tissue responds to physical activity, exercise and disuse in COPD? Inflammation is a complicated tangled web of intertwining cascades that are carefully controlled by numerous positive and negative feedback and feed-forward mechanisms. Until we develop a better understanding of the influence of inflammation on muscle injury, regeneration and healing in COPD, clinicians should strongly encourage their COPD patients to stop smoking, exercise regularly and engage in pulmonary rehabilitation, which are proven ways to enhance muscle performance in COPD patients.

\section{Competing interests: None.}

Thorax 2008;63:95-96. doi:10.1136/thx.2007.088575

\section{REFERENCES}

1. Decramer M, Gosselink R, Troosters T, et al. Muscle weakness is related to utilization of health care resources in COPD patients. Eur Respir J 1997;10:417-23.

2. Swallow EB, Reyes D, Hopkinson NS, et al. Quadriceps strength predicts mortality in patients with moderate to severe chronic obstructive pulmonary disease. Thorax 2007;62:115-20.

3. Wouters EF. Chronic obstructive pulmonary disease. 5 systemic effects of COPD. Thorax 2002;57:1067-70.

4. Broekhuizen R, Wouters EF, Creutzberg EC, et al. Raised CRP levels mark metabolic and functional impairment in advanced COPD. Thorax 2006;61:17-22.

5. Yende S, Waterer GW, Tolley EA, et al. Inflammatory markers are associated with ventilatory limitation and muscle dysfunction in obstructive lung disease in well functioning elderly subjects. Thorax 2006;61:10-16.

6. Langen RC, Schols AM, Kelders MC, et al. Muscle wasting and impaired muscle regeneration in a murine model of chronic pulmonary inflammation. Am J Respir Cell Mol Biol 2006;35:689-96.

7. Barreiro E, Schols AMWJ, Polkey Ml, et al. Cytokine profile in quadriceps muscles of patients with severe chronic obstructive pulmonary disease. Thorax 2008;63:100-7.
8. Chen SE, Jin B, Li YP. TNF-alpha regulates myogenesis and muscle regeneration by activating p38 MAPK. Am J Physiol Cell Physiol 2007;292:C1660-71.

9. Tayek JA. Effects of tumor necrosis factor alpha on skeletal muscle amino acid metabolism studied invivo. J Am Coll Nutr 1996:15:164-8.

10. Contreras-Shannon V, Ochoa 0, Reyes-Reyna SM, et al. Fat accumulation with altered inflammation and regeneration in skeletal muscle of CCR2-/- mice following ischemic injury. Am J Physiol Cell Physiol 2007;292:C953-67.

11. Alvarez B, Quinn LS, Busquets $S$, et al. Direct effects of tumor necrosis factor alpha (TNF-alpha) on murine skeletal muscle cell lines. Bimodal effects on protein metabolism. Eur Cytokine Netw 2001;12:399410.

12. Montes de Oca M, Torres SH, De Sanctis J, et al. Skeletal muscle inflammation and nitric oxide in patients with COPD. Eur Respir J 2005;26:390-7.

13. Gosker HR, Wouters EF, van der Vusse GJ, et al. Skeletal muscle dysfunction in chronic obstructive pulmonary disease and chronic heart failure: underlying mechanisms and therapy perspectives. Am J Clin Nutr 2000:71:1033-47.

14. Anker SD, Coats AJ. How to RECOVER from RENAISSANCE? The significance of the results of RECOVER, RENAISSANCE, RENEWAL and ATTACH. Int J Cardiol 2002;86:123-30.

15. Chung ES, Packer M, Lo KH, et al. Randomized, double-blind, placebo-controlled, pilot trial of infliximab, a chimeric monoclonal antibody to tumor necrosis factor-alpha, in patients with moderate-tosevere heart failure: results of the anti-TNF Therapy Against Congestive Heart Failure (ATTACH) trial. Circulation 2003;107:3133-40.

16. Marsolais D, Duchesne $\mathrm{E}$, Cote $\mathrm{CH}$, et al. Inflammatory cells do not decrease the ultimate tensile strength of intact tendons in vivo and in vitro: protective role of mechanical loading. J Appl Physiol 2007; 102:11-17.

\section{Lung clearance index in CF: a sensitive marker of lung disease severity}

\section{Jane C Davies, 1,2,3 Steve Cunningham, ${ }^{3,4}$ Eric W F W Alton, ${ }^{1,3,5} \mathrm{~J}$ A Innes I,6 $^{3,6}$}

Despite airways which are thought to be normal at birth, $90 \%$ of patients with cystic fibrosis (CF) ultimately die from respiratory complications of the disease. The steps involved in the progressive

\footnotetext{
${ }^{1}$ Department of Gene Therapy, Imperial College, London, UK; ${ }^{2}$ Department of Paediatric Respiratory Medicine, Royal Brompton Hospital, London, UK; ${ }^{3}$ UK Cystic Fibrosis Gene Therapy Consortium, UK; ${ }^{4}$ Department of Paediatric Respiratory Medicine, Hospital for Sick Children, Edinburgh, UK; ${ }^{5}$ Department of Respiratory Medicine, Royal Brompton Hospital, London, UK;

${ }^{6}$ Respiratory Unit, Western General Hospital, Edinburgh, UK
}

Correspondence to: Dr J C Davies, Department of Gene Therapy, Imperial College, London, UK; j.c.davies@ imperial.ac.uk destruction of the airways, and strategies aimed at limiting these processes, are therefore major areas of research. For both research and clinical purposes, measures of lung involvement should ideally be (1) sensitive enough to detect abnormalities early and directly reflect changes in disease severity, either naturally occurring or in response to interventions; (2) feasible and reproducible in all age groups; and (3) repeatable over time.

With regard to the first of these criteria, there is increasing concern that conventional measures such as spirometry and chest radiography are insufficiently sensitive, particularly at the mild and moderate stages of disease. Use of these investigations has enabled a generation of clinicians to improve pulmonary status significantly, but these clinical improvements themselves serve to highlight the inadequacy of the tools we have available to assess them. In contrast to the situation several decades ago, forced expiratory volume in $1 \mathrm{~s}\left(\mathrm{FEV}_{1}\right)$ now falls too late and too slowly to be accepted unquestioningly as the gold standard. From bronchoscopic studies there is growing concern that, by the time routine spirometry is abnormal, a self-perpetuating infective and inflammatory process has taken hold within the lung that may be difficult to reverse. ${ }^{12}$ Furthermore, reports of annual falls in $\mathrm{FEV}_{1}$ as low as $1 \%^{34}$ mean that any new intervention aimed, early in the course of the disease, at slowing this decline will require huge numbers of patients and long duration studies to achieve sufficient power. Similarly, plain radiographs fail to detect early changes, although reflect relatively well the gross anatomical abnormalities of end-stage disease. With regard to the second criterion (applicability across the age range), infant lung function is being pioneered by an increasing number of 